\title{
A GEOMETRIC VARIANT OF BADE'S THEOREM ON DOMINATING MEASURES
}

\author{
MAHLON M. DAY
}

\begin{abstract}
Let $\mathscr{B}$ be a bounded Boolean algebra of projections in a superreflexive Banach space $B$. Then for each $b$ in $B$ there is a $\beta=\varphi(b)$ in $B^{*}$ such that $\varphi$ is norm-norm uniformly bicontinuous and $\beta(P b)=0$ if and only if $P b=0$.
\end{abstract}

1. Introduction. Bade (1955) showed that if $B$ is a Banach space and if $\mathscr{B}$ is a complete Boolean algebra of projections of $B$ into itself, then for each $b$ in $B$ there is a dominating element $\beta$ in $B^{*}$ such that for each $P$ in $\mathscr{B}, \beta(P b)=0$ if and only if $P b=0$. From a measure-theoretic point of view, setting $\alpha(P)=\beta(P b)$ gives a positive measure $\alpha$ on $\mathscr{B}$ such that the vector measure $P b$ from $\mathscr{B}$ into $B$ is absolutely continuous with respect to $\alpha$.

Bade's proof requires completeness of $\mathscr{B}$ but asks nothing special of the space $B$; his proof uses the Stone representation space of $\mathscr{B}$. The present proof uses geometric methods which require strong hypotheses on the Banach space but ask only boundedness of $\mathscr{B}$. Also, this method gives continuous dependence of $\beta$ on $b$.

2. Geometry of the unit ball in a Banach space. Let $U$ and $S\left[U^{\pi}\right.$ and $\left.S^{\prime}\right]$ be the unit ball and unit sphere in $B$ [in $B^{*}$ ]. Define $J$ from $S$ to $S^{\prime}$ by: For each $b$ in $S$, $J b=\left\{\beta\right.$ in $\left.S^{\prime} \mid \beta(b)=1\right\}$. For the following facts about $J$ see, for example, Cudia (1964).

LemMA 2A. (i) For each $b$ in $S, J b$ is $a w^{*}$-closed convex subset of $S^{\prime}$.

(ii) $\mathrm{Jb}$ is a singleton $\{\beta\}$ if and only if the Gateaux derivative of \|\| exists at $b$ and is $\beta$.

(iii) When the norm topology is used in $S$ and in $S^{\prime}, J$ is [uniformly] continuous on $S$ if and only if \|\| is [uniformly] Fréchet differentiable on $S$.

LEMMA 2.1. If $\mathscr{B}$ is a Boolean algebra of projections in $B$, each of norm $<1$, if $b$ is in $S$, and if a point $\beta$ in $J b$ exposes $b$ on $U$, then $\beta(P b)=0$ implies that $P b=0$.

Proof. If $\beta(P b)=0$, then $\beta(I-P)(b)=\beta(b)=1$. But $\|(I-P)(b)\|<1$; that is, $(I-P)(b)$ is in $U \cap \beta^{-1}(1)=\{b\}$, because $\beta$ exposes $b$ on $U$. Hence $(I-P)(b)=b$ or $P b=0$.

COROLlaRY 2.2. If $B$ is rotund and if $\mathscr{B}$ is a Boolean algebra of projections in $B$ of norm $\leqslant 1$, then each $\beta$ in $J b$ satisfies the condition that $\beta(P b)=0$ implies that $P b=0$.

Received by the editors March 21, 1980.

1980 Mathematics Subject Classification. Primary 46B20, 47D05, 47D10; Secondary 28 C99.

(c) 1981 American Mathematical Society 0002-9939/81/0000-0161/\$01.75 
This is a consequence of the well-known fact that $B$ is rotund if and only if every point of $S$ is an exposed point of $U$. (See, for example, Day (1958 or 1973), VII, 2(1).)

CoROllary 2.3. If \|\| in $B$ is rotund and [uniformly] Fréchet differentiable and if $\mathscr{B}$ is a Boolean algebra of projections in $B$ of norm $<1$, then $J$ is [uniformly] norm-norm continuous from $S$ into $S^{\prime}$ and $[\mathrm{Jb}](\mathrm{Pb})=0$ if and only if $\mathrm{Pb}=0$.

For proof use Lemma 2A, (ii) and (iii), and Lemma 2.1.

3. Renorming. Let $\mathscr{B}$ be a bounded Boolean algebra of projections in $B$. For each $P$ in $\mathscr{B}$ define $V=2 P-I$. Then $V$ is an involution and $\mathcal{V}=\{V \mid V=2 P-$ $I$ and $P \in \mathscr{B}\}$ is a bounded abelian group of involutions of $B$ onto $B$.

For any bounded group $\mathfrak{V}$ of isomorphisms of $B$ onto $B$ it is easy to define at least one norm on $B$ isomorphic to the original norm and invariant under all elements of the group; let

$$
p_{1}(b)=\sup \{\|V b\|, V \in \mathfrak{V}\}
$$

Clearly, $\|P\|_{p_{1}}=\|V+I\|_{p_{1}} / 2 \leqslant 1$ for each $P$ in $\mathscr{B}$. The difficulty is that this new norm may have lost whatever roundness or smoothness properties the original norm may have had. (A picture of two smooth bathtubs intersecting in a square shows one possibility when $B$ is two-dimensional and $\mathcal{V}$ has one reflection.) We must either renorm in a completely different way to avoid this or else renorm again to improve the norm and maintain the isometry property of each $V$.

If $B$ is a complete inner-product space, a different construction (Sz. Nagy (1947); Dixmier (1950); Day (1957), p. 542) avoids the difficulty by using the amenability of each abelian group; this was proved, but not so named, by von Neumann (1929).

THEOREM 3.1. If $H$ is a complete inner-product space and if $\mathscr{B}$ is a bounded Boolean algebra of projections in $H$, then there is a new isomorphic inner product [,] in $H$ under which each $V$ in $\mathscr{V}$ is an isometry, $[P x, x]=0$ if and only if $P x=0$, and under which $J x=[, x]$ is a uniformly continuous function of $x$ on $S$.

Proof. Let ( , ) be the inner-product attached to the original norm in $H$. Let $\alpha$ be any invariant mean on the bounded functions on $\mathfrak{V}$. For each $x, y$ in $H$ let $f_{x, y}(V)=(V x, V y)$ for all $V$ in $\mathcal{T}$, and let $[x, y]=\alpha\left(f_{x, y}\right)$. It is easily seen that this inner-product determines a $\mathfrak{V}$-invariant norm isomorphic to the original norm. Also for $x$ and $y$ in $S,[y, x]=[J x](y)$, so Corollary 2.3 can be used.

If $B$ is not a Hilbert space but is superreflexive, much the same result is attainable by different methods. The next lemma is from my paper Invariant renorming, Day (1976). The properties of superreflexivity used can be found in Day (1973), Chapter VII, §4, B, especially, Theorem 4.

LEMMA 3A. If $B$ is superreflexive and if $G$ is a group of linear isometries of $B$ onto $B$, then there is a new isomorphic norm in $B$ such that the new space is uniformly convex and the elements of $G$ are still isometries.

(To prove this merely follow the proof of Enflo (1972) of renormability of $B$, observing that each step is invariant under all elements of $G$.) 
Theorem 3.2. Let $B$ be a superreflexive space and let $\mathscr{B}$ be a bounded Boolean algebra of projections in $B$. Then there is an isomorphic uniformly convex and uniformly Fréchet differentiable norm in $B$ for which all $P$ in $\mathscr{B}$ are of norm $<1, J$ is uniformly continuous, as is $J^{-1}$, and $[J b](P b)=0$ if and only if $P b=0$, and $\left[P^{*} \beta\right]\left(J^{-1} \beta\right)=0$ if and only if $P^{*} \beta=0$.

Proof. As before, construct $\mathscr{V}=\{V=2 P-I \mid P \in \mathscr{B}\}$. Then construct the $\mathfrak{V}$-invariant norm $p_{1}$, defined in line 8 of this section. The space with norm $p_{1}$ is still superreflexive but the elements of $\mathcal{V}$ are now isometries. Use Lemma 3A with $\mathcal{V}$ and $p_{1}$ to get a new uniformly convex norm $p_{2}$ isomorphic to $p_{1}$ and still invariant under $\mathcal{V}$. Use Lemma $3 A$ again with $\mathcal{V}^{*}$ and $p_{2}^{*}$ in the superreflexive conjugate space $B^{*}$ to get a uniformly convex norm $p_{3}^{*}$ in $B^{*}$; then $p_{3}^{*}$ is conjugate to some norm $p_{3}$ in $B$ and $p_{3}$ will also be $\mathfrak{V}$-invariant. Construct the Asplund (1967) average of $p_{2}$ and $p_{3}$ and call it $p_{4}$. Then $p_{4}$ will be both uniformly rotund and uniformly smooth, and will also be invariant under $\mathfrak{T}$. By Corollary $2.3, J$ is uniformly continuous and $[J b](P b)=0$ if and only if $P b=0$. Since $J^{-1}$ is just the corresponding map defined from $B^{*}$ onto $B, J^{-1}$ is also uniformly continuous, and $\left[P^{*} \beta\right]\left(J^{-1} \beta\right)=0$ if and only if $P^{*} \beta=0$.

COROllary 3.3. If $\mathscr{B}$ is a bounded Boolean algebra in a superreflexive space $B$, then there is a biuniformly continuous homeomorphism $\Phi$ of $S$ onto $S^{\prime}$ such that for each $P$ in $B,[\Phi b](P b)=0$ if and only if $P b=0$, and also $\left[P^{*} \beta\right]\left(\Phi^{-1} \beta\right)=0$ if and only if $P^{*} \beta=0$.

Proof. Temporarily, let $S$ and $\Sigma\left[S^{\prime}\right.$ and $\left.\Sigma^{\prime}\right]$ be unit spheres for \|\| and for $p_{4}$ [for \|\|$^{*}$ and for $p_{4}{ }^{*}$ ]. Define the radial maps $\rho(b)=b / p_{4}(b)$ from $S$ onto $\Sigma$, and $r(\beta)=\beta /\|\beta\|^{*}$ from $\Sigma^{\prime}$ onto $S^{\prime}$. These functions are biuniformly continuous (in fact, Lipschitz) homeomorphisms. If $\Phi(b)=r(J(\rho(b)))$, then $\Phi$ is uniformly continuous from $S$ onto $S^{\prime}$, and $\Phi^{-1}=\rho^{-1} J^{-1} r^{-1}$ is also uniformly continuous from $S^{\prime}$ onto $S$. Thus $\Phi$ also has the other properties of the ccrollary.

\section{BIBLIOGRAPHY}

E. Asplund (1967), Averaged norms, Israel J. Math. 5, 227-233.

W. Bade (1955), On Boolean algebras of projections and algebras of operators, Trans. Amer. Math. Soc. 80, 345-360.

D. F. Cudia (1964), The geometry of Banach spaces: Smoothness, Trans. Amer. Math. Soc. 110, 284-314.

M. M. Day (1958, revised 1973), Normed linear spaces, Springer-Verlag, Berlin, Heidelberg and New York.

(1957), Amenable semigroups, Illinois J. Math. 1, 509-544.

(1976), "Invariant renorming" in Fixed point theory and its applications, (S. Swaminathan, Ed.), Academic Press, New York, San Francisco and London.

J. Dixmier (1950), Les moyennes invariantes dans les semigroupes et leurs applications, Acta Sci. Math. (Szeged) 12, 213-227.

P. Enflo (1972), Banach spaces which can be given an equivalent uniformly convex norm, Israel J. Math. 13, 281-288.

J. von Neumann (1929), Zur allgemeine Theorie des Masses, Fund. Math. 13, 73-116.

B. Sz. Nagy (1947), On uniformly bounded linear transformations in Hilbert space, Acta Univ. Szegediensis Sect. Sci. Math. 11, 152-157.

Department of MAthematics, University of Illinois, Urbana, Illinois 61801 